\title{
Educación bilingüe y actitudes lingüísticas en Els Ports (Castellón) y Matarranya (Teruel)
}

\author{
Juan González Martínez \\ Rebut: 22/02/2012 Acceptat: 28/11/2012
}

\begin{abstract}
Resumen
El análisis de las actitudes hacia el catalán y hacia la educación bilingüe en las vecinas comarcas de Els Ports (Castellón) y Matarranya (Teruel), continúa demostrando que en ambas comarcas catalán y español conviven todavía hoy en una situación de diglosia. Sin embargo, es sencillo documentar una diferente percepción acerca de la presencia del catalán en el sistema educativo entre los habitantes de Els Ports y los de Matarranya. En esta investigación, por medio de la técnica de la entrevista sociolingüística, intentamos sondear, precisamente, cuáles son esas actitudes hacia la educación bilingüe, en catalán y en castellano. Tras ese análisis, no queda ninguna duda de que las actitudes son mucho menos positivas en Matarranya que en Els Ports. Como consecuencia de ello, concluimos que la puesta en marcha de políticas educativas bilingües, como las que rigen en otras zonas catalanohablantes, se configura como una de las soluciones para mejorar la situación sociolingüística de Matarranya.
\end{abstract}

Palabras clave: actitudes lingüísticas, educación bilingüe, diglosia, catalán, español

\section{Bilingual education and linguistic attitudes in Els Ports (Castellón) and Matarranya (Teruel)}

\section{Abstract}

An analysis of the attitudes towards Catalan and the bilingual education in the adjacent regions of Els Ports (in the province of Castellón, Spain) and Matarranya (in the province of Teruel, Spain) shows that in both areas, Catalan and Spanish coexist today in a diglossic situation, but we can easyly document a very different perception of the presence of Catalan in the educational system between the inhabitants from

\footnotetext{
${ }^{a}$ Departament de Pedagogia. Universitat Rovira i Virgili - Tarragona
} 
Els Ports or Matarranya. By this research, and through sociolinguistic interviews, we try to assess language attitudes towards bilingual education, both in Catalan and Spanish. After that analysis, we have no doubt that those attitudes are less possitive in Matarranya than in Els Ports. As a consequence of this, we consider that bilingual education policies, as those we can find in other Catalan-speaking areas, are the best solucion for improving the sociolinguistic status in Matarranya.

Keywords: language attitudes, bilingual education, diglossia, Catalan, Spanish

\section{Introducción}

Pese a no tratarse de una nueva realidad, el interés por la educación bilingüe viene siendo frecuente en las regiones catalanohablantes, especialmente en los últimos años; y no solo desde el punto de vista de la necesaria internacionalización de los sistemas educativos, sino también desde perspectivas que intentan afrontar los retos inherentes a contextos educativos caracterizados por movimientos migratorios o por la existencia de comunidades de habla bilingües por naturaleza -o incluso ambas realidades solapadas-, en los cuales puede rastrearse con facilidad la presencia de dos o más variedades lingüísticas de uso habitual. En todos esos casos, es habitual que los alumnos se vean abocados al aprendizaje de dos o más sistemas en el entorno escolar. Sin duda, ello es particularmente pertinente al referirnos a los territorios bilingües, en los que el alumnado aprende -o debería aprender- las dos lenguas de uso habitual en su comunidad de habla, y a las que hay que añadir los denominados idiomas internacionales (inglés, francés, español, etc.).

Debemos partir de la premisa de que resulta muy complejo poder clasificar las consecuencias educativas de ese bilingüismo tan extendido del que empezábamos hablando; sin embargo, la literatura nos provee de diferentes modelos para este fin. Y a partir de ellos, se ha intentado recoger cuáles son las variables que más contribuyen a la conformación del resultado del programa educativo: cuál es la posición social de la lengua de los estudiantes, cuál es la lengua de instrucción o cuáles son los objetivos lingüísticos del programa educativo bajo el cual se escolarizan los estudiantes, por ejemplo (y así lo convienen muchos de los autores que se han dedicado a reflexionar sobre estos aspectos, tanto en el panorama nacional como en el internacional: Mackey, 1970 y 1976; Siguan y Mackey, 1986; Skutnabb-Kangas, 1988; Genesse, 2006; Lindholm-Leary, 2006; Huguet et al., 2008).

La diferente situación de una comunidad de habla con respecto de estas variables, en principio, señala la existencia de tipos diferentes de programas: de segregación, de subversión, de mantenimiento de inmersión y de inmersión propiamente dicha (Arnau, 1992; Huguet et al., 2008). Y se acepta comúnmente que los diferentes modelos tienen consecuencias directas también diferentes, pues solo los programas de mantenimiento y de inmersión plena consiguen el objetivo de promover el bilingüismo real $y$, un paso más, el biculturalismo del alumnado. Por el contrario, los programas educativos basados en la segregación y la subversión dificultan el 
desarrollo de la competencia comunicativa en todas las variedades presentes en el sistema educativo y conducen indefectiblemente a un bajo rendimiento académico (Huguet y Llurda, 2001: 268).

Aunque no exentos de polémica, los programas de inmersión lingüística han gozado de considerable predicamento desde los primeros experimentos de la escuela de Lambert en Montreal (Lambert y Tucker, 1972; Lambert, 1974; Genesee, 1986). Desde entonces y en adelante, se viene sosteniendo que la participación de los estudiantes en programas de este tipo no les ocasiona pérdida alguna ni en su cultura ni en su lengua materna; y que, bien al contrario, reportan considerables beneficios y contribuyen a la consolidación del bilingüismo en las sociedades en las que se aplican. España, sin duda, no ha sido una excepción en el diseño de programas de inmersión lingüística, y de ello son buena muestra los modelos aplicados en el País Vasco y Cataluña, plenamente consolidados (Siguan, 1992; Vila, 1995, 1998; Ferrer, 2000; Torres-Guzmán y Etxeberría, 2005; Huguet, 2006, 2007; Rendon, 2007), si bien en ambos casos dicha inmersión merece algunas precisiones -por cuanto, en general, la inmersión solo se aplica estrictamente con perfiles concretos de alumnos, y no en el modelo educativo general.

En oposición a ello, contamos en el territorio peninsular con comunidades de habla para las cuales, a pesar de su condición bilingüe, no han sido aplicadas estas mismas políticas lingüísticas. Ejemplo de ello podrían serlo perfectamente la Comunidad Valenciana y las zonas catalanohablantes de Aragón, también muy diferentes entre sí, como veremos. Si bien ambos territorios forman parte del dominio lingüístico catalán, la situación es allí bien diferente por lo que respecta a la convivencia de las variedades española y catalana en general, y en cuanto a la consideración oficial que estas variedades reciben en particular. Y así, en la Comunidad Valenciana la modalidad propia del catalán, conocida como valenciano, es cooficial con el español; y de ahí que esté garantizada su presencia en cualquier situación social y que, con una diferente perspectiva a la de Cataluña, se impulsen políticas que promuevan su uso social (por medio de la educación o de los medios de comunicación, entre otros). Por el contrario, Aragón es una comunidad oficialmente monolingüe -a falta de la concreción de la Ley de Lenguas de Aragón de 2009,1 tan esperada por los territorios bilingües-; $y$, por tanto, en esos territorios no se garantiza el uso social de las modalidades propias de catalán o de fabla aragonesa ni, casi podríamos añadir, se permite su uso en instancias oficiales. Hablamos, por supuesto, de la estrecha franja de territorio limítrofe con Cataluña, en las provincias de Huesca, Zaragoza y Teruel, que se conoce como Franja Oriental de Aragón (o, en su denominación catalana, Franja de Ponent), pertenecientes al dominio lingüístico catalán; o de zonas del Pirineo donde todavía pervive la recién mencionada fabla aragonesa.

Si bien el futuro inmediato del catalán en la Comunidad Valenciana dista mucho de estar garantizado ( $y$ numerosas voces claman por su evidente retroceso y, por consiguiente, por el peligro inminente de su desaparición), según el informe de la Acadèmia Valenciana de la Llengua (2004) esta es la lengua que usa preferente o indistintamente entre el $40 \%$ y el $45 \%$ de la población del territorio bilingüe, y un 
76\% de los habitantes de la Comunidad Valenciana se siente con bastante o mucha competencia en ella; y, además, es la lengua de instrucción (o una de ellas) de gran parte de los niños de la escuela, y tiene el apoyo oficial y la protección del Govern de la Generalitat Valenciana. Por el contrario, los territorios catalanohablantes de la Franja Oriental de Aragón no han sido destino de intento alguno de revitalización del uso y de la presencia del catalán (más allá de episodios que casi podríamos considerar folkloristas por parte de algunos consejos comarcales), la lengua no está oficialmente reconocida y queda recluida, pues, en la esfera de lo privado, a pesar de que alrededor de un $80 \%$ de la población es hablante nativa de catalán; y todo ello, en pleno contraste con el español, lengua oficial, lengua formal y lengua de instrucción en la enseñanza.

$\mathrm{Ni}$ que decir tiene que, si las diferencias son ya de hondo calado en la política lingüística general, más notables aún resultan si atendemos al tratamiento del bilingüismo social en el sistema educativo. Y así, si en Cataluña hablábamos de la puesta en marcha de políticas que consideran la pertinencia de modelos de inmersión lingüística, en la Comunidad Valenciana hablaríamos mejor de programas pluralistas de mantenimiento de la inmersión lingüística; en ellos, con desigual grado, lo que se pretende es ir asimilando a cuantos más alumnos al uso habitual del valenciano, por medio de diferentes programas educativos que combinan mayor o menor presencia de la lengua propia como sistema lingüístico vehicular del aprendizaje (como el PIL, Programa de Immersió Lingüística; o el PEV, Programa de Ensenyament en Valencià; cuyo objetivo es siempre garantizar la competencia comunicativa en valenciano del alumnado). En clara oposición, en las comarcas catalanohablantes de la Franja Oriental de Aragón, lo que vamos encontrando son diferentes propuestas en las que el catalán no recibe tratamiento alguno en las aulas, o en las que pasa a convertirse en una opción de pocas horas a la semana que los centros pueden ofertar y que los padres del alumnado pueden elegir para sus hijos (Huguet et al., 2008); esto es, algo muy lejano de una política lingüística educativa con verdadero afán de promoción de la lengua minoritaria y, por tanto, con un efecto previsiblemente anecdótico.

Como sabemos, las diferencias entre lo que sucede a este respecto entre los territorios contiguos de Aragón y Cataluña han sido objeto de estudio de modo profuso. En este sentido, pensamos, como no podía ser de otro modo, en el reiterado interés que han venido suscitando las comarcas vecinas de Baix Cinca (Huesca, Aragón) y Baix Segre (Lleida, Cataluña) para Huguet, quien ha abordado su estudio en diferentes ocasiones (Huguet y Biscarri, 1995; Huguet y Llurda, 2001; Huguet, 2006; Huguet et al., 2008, etc.). Y nos resulta de especial interés la aportación de Huguet y Llurda (2005), por cuanto incide en el componente actitudinal que acompaña a esas diferentes políticas lingüísticas, aplicadas a uno y otro lado de la frontera catalanoaragonesa en ese punto. No en vano, y aun a riesgo de resumir en exceso, al analizar las diferencias entre ambos escolares, Huguet llega a conclusiones tan interesantes como asimilar, por favorables, las actitudes de los informantes oscenses que asisten con regularidad a clases de catalán (aun desde un enfoque 
asimilacionista segregador) y de sus vecinos catalanes (bajo un modelo pluralista de inmersión). Y lo mismo se ratifica en Huguet, Lapresta y Madariaga (2008), en su análisis de las actitudes lingüísticas no solo hacia las lenguas presentes en dichas comunidades de habla, sino también con respecto de las extranjeras incluidas en el sistema educativo. Por último, en sentido parecido apuntan las conclusiones de más recientes investigaciones acerca de las actitudes lingüísticas de los escolares hacia sistemas bilingües de Cataluña (Lapresta, Huguet y Janés, 2010) o el País Vasco (Rojo, Madariaga y Huguet, 2010).

Sin embargo, hasta donde conocemos, no es frecuente el análisis comparativo entre estas dos realidades, a uno y otro lado de la frontera valenciano-aragonesa; y, nosotros mismos, en anteriores investigaciones, nos hemos centrado más en el componente actitudinal inherente a esa realidad sociolingüística, y no específicamente a lo relativo a la educación bilingüe (González Martínez y Blas Arroyo, 2012). Sin embargo, no tenemos dudas acerca de lo provechoso de ese análisis comparativo, especialmente enfocado desde el punto de vista de las actitudes no solo hacia las propias variedades lingüísticas, sino también hacia su presencia en el sistema educativo, motivo por el cual le dedicamos esta reflexión que nos ocupa ahora.

El presente trabajo, pues, se propone realizar un análisis de las actitudes lingüísticas hacia el catalán y hacia su presencia en el sistema educativo en dos comarcas vecinas representativas de cuanto sucede a ambos lados de la frontera valenciano-aragonesa y dentro del dominio lingüístico catalán: Els Ports (en el norte de la provincia de Castellón, Comunidad Valenciana) y Matarranya (limítrofe con la anterior, ya en Teruel, Aragón).

\section{Las comunidades de habla estudiadas}

Las comunidades de habla que analizamos desde la perspectiva de las actitudes hacia la educación bilingüe son Els Ports (Castellón) y Matarranya (Teruel). 


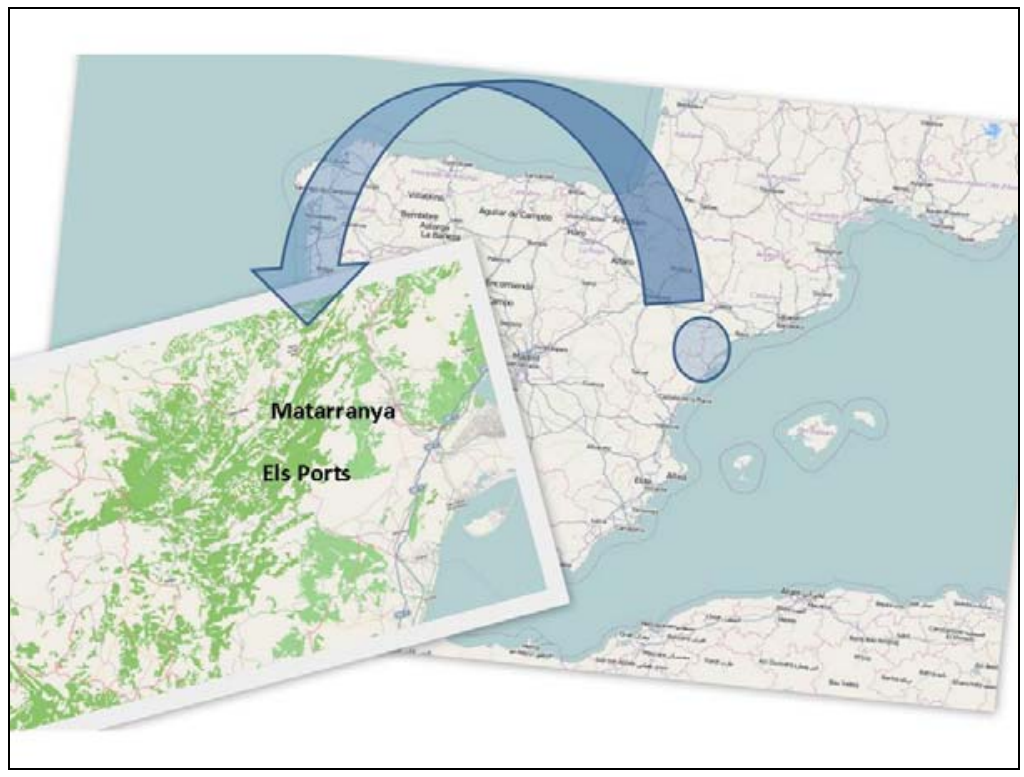

Imagen 1. Mapa de las comarcas de Els Ports y Matarranya.

A pesar de su cercanía y de su innegable parecido demográfico, las dos comunidades son sustancialmente diferentes desde una perspectiva sociolingüística, y eso es bien evidente por lo que respecta al tratamiento que reciben el catalán y el castellano en la educación obligatoria. En Els Ports, la política lingüística para la promoción del catalán se materializa en una presencia garantizada de esta lengua en la realidad educativa (a partir del desarrollo de la Llei d'Ús i Ensenyament del Valencià de 1983). En cambio, en la comarca de Matarranya, la modalidad catalana vernácula no recibe ningún reconocimiento oficial $y$, por consiguiente, es el español la única lengua que se considera en la enseñanza obligatoria (si bien en con desigual grado y diferente regularidad, los alumnos tienen la oportunidad de cursar una asignatura optativa de catalán durante la Educación Secundaria Obligatoria); además, en los medios de comunicación oficiales, tampoco está presente modalidad alguna de catalán. Para completar el cuadro, la comarca de Matarranya es la única de la provincia de Teruel en la que se da una situación de contacto de lenguas. Así, es plausible pensar que la diglosia de la que, históricamente, también se partía en la comarca de Els Ports siga vigente en todo el espectro social de la comarca turolense (es decir, no solo en las generaciones mayor y adulta, sino también en la generación joven, aquella en la que la acción de las políticas educativas podría empezar a notarse), como así parecen indicar los datos obtenidos en anteriores investigaciones González Martínez (2010, 2011) y González Martínez y Blas Arroyo (2011, 2012). 


\section{Objetivo}

En el contexto inicial ya referido, los objetivos principales de esta investigación son: (1) plantear, por medio de una entrevista semidirigida con preguntas abiertas y cerradas, un análisis de las actitudes lingüísticas de los hablantes de las comarcas bilingües de Els Ports (Castellón) y Matarranya (Teruel) hacia la presencia del catalán lengua propia, de uso habitual, en ambas- en el sistema educativo, en el contexto de la histórica situación de diglosia de su convivencia con el español; y (2) atender a la posible incidencia de las políticas lingüísticas en la conformación de las actitudes lingüísticas de los jóvenes, que deberán ser más positivas hacia la lengua propia en Els Ports, que en Matarranya, como resultado de la diferente situación sociolingüística general de ambas comunidades de habla.

\section{Metodología e instrumentos}

Como complemento a otras investigaciones llevadas a cabo en estos mismos territorios (González Martínez, 2010, 2011; González Martínez y Blas Arroyo, 2011, 2012), nuestra investigación acerca de las actitudes lingüísticas en ambas comarcas se ha llevado a cabo por medio de un cuestionario sociolingüístico contextualizado dentro de una entrevista semidirigida, técnica que, en nuestra opinión, ofrece múltiples ventajas: (1) los cuestionarios permiten un análisis de los datos siempre exhaustivo y revisable; (2) ayudan al establecimiento de indicadores con los que valorar mejor la situación sociolingüística de la comunidad de habla (por ejemplo, los parámetros de fidelidad u orgullo lingüísticos analizados en estas páginas); y (3) facilitan la comparación con otras investigaciones ya realizadas, bien de la misma comunidad, bien de otras cercanas. ${ }^{2}$

Una parte de este cuestionario sondeaba, específicamente, lo que se ha dado en llamar comúnmente fidelidad lingüística, en directa relación con nuestro objetivo de analizar las actitudes hacia la escuela bilingüe en estos dos territorios. Veamos. Si entendemos la fidelidad lingüística como la resistencia de los hablantes a la pérdida de usos sociales de la propia lengua (en palabras de Weinreich, 1953), esta se puede valorar por medio de una serie de preguntas relacionadas con el uso potencial de esa lengua en dominios sociolingüísticos formales, generalmente la educación; en efecto, así lo propone este autor en su definición fundacional. En nuestro caso, tomamos como referencia otras investigaciones similares a la presente (Blas Arroyo, 1994; González Martínez, 2010), de tal modo que este indicador se obtiene, en efecto, a partir de las respuestas a tres preguntas relacionadas con: a) la enseñanza del catalán en educación primaria y secundaria; b) la enseñanza en catalán en ambos niveles, y c) la enseñanza en catalán en la Universidad. Y los enunciados de esas tres preguntas, debidamente validados, eran los siguientes: a) ¿Es importante que se enseñe el catalán en las escuelas?; b) ¿Es bueno que se enseñe en catalán en las escuelas y en los institutos?; c) Y en la Universidad, ¿qué le/te parece que se enseñe en catalán? Y las respuestas se organizaban en una escala con los siguientes valores: 1 Por supuesto, 
Muy bien; 2 Sí, Bien; 3 Ni sí ni no, Ni bien ni mal; 4 No, mal, Mal; 5 En absoluto, Muy mal.

Estos enunciados se completaban con el contraste con otros indicadores como el índice de orgullo lingüístico o el índice de presión social subjetiva. Ambos escapan de modo directo al propósito de esta reflexión actual, por lo que nos referiremos a ellos sucintamente. Sin embargo, puede ampliarse la información sobre ellos en González Martínez (2010, 2011). A título de reseña, diremos que el orgullo lingüístico lo entendemos como el entusiasmo que los hablantes sienten por la propia lengua (Weinreich, 1953). Siguiendo la forma habitual de analizarlo en nuestro contexto (Blas Arroyo, 1994; González Martínez, 2010), en el caso de nuestra comunidad de habla lo escrutamos por medio de dos preguntas (sobre la obligatoriedad -al menos moralque los inmigrantes de la comarca deberían tener a la hora de aprender la lengua propia, y acerca de las opiniones de los informantes sobre el requisito lingüístico que opera en el acceso a la función pública en las Comunidades Autónomas con lengua propia). Por su parte, el índice de presión social subjetiva concreta ese interés general por la defensa de la propia lengua del que hablábamos antes en el «entusiasmo que los hablantes manifiestan ante el empleo de la lengua propia [en este caso, el catalán] en determinados registros oficiales de especial relevancia» (Blas Arroyo, 1994: 147). En nuestro estudio, evaluamos esa presión social subjetiva en la obligación de usar el valenciano en actos oficiales y en los medios de comunicación.

\subsection{Informantes y procedimiento}

Este cuestionario sociolingüístico se distribuyó a 30 informantes de Els Ports y otros 30 de Matarranya, que conformaban nuestra muestra empírica o razonada (López Morales 1994), obtenida por medio de una técnica de muestreo seleccionado o predeterminado (Silva-Corvalán 1989: 18); en ella, se establecieron diferentes subgrupos en función de los tres factores sociales que hemos analizado: género, edad y estrato social, de los que ahora nos interesa especialmente el factor generacional (de nuevo, por limitaciones en el espacio, solo glosaremos los resultados del factor social edad; en relación con la incidencia de los factores de género y estrato social, puede consultarse, de nuevo, González Martínez (2010, 2011).

Con todo, más relevancia tiene para los objetivos de la investigación reseñados más arriba (véase, apartado 2), evaluar la incidencia del factor generacional, que establecemos en nuestro estudio por medio de tres grandes grupos (1. ${ }^{a}$ generación o jóvenes, de 17 a 25 años;.$^{a}$ generación o adultos, de 35 a 50 años; y $3 .^{a}$ generación o mayores, por encima de los 60 años). Para que -como sospechamos- pueda hablarse de dinamismo en la situación (socio)lingüística de la comunidad de habla, este debe apreciarse en una diferencia significativa entre las actitudes lingüísticas de los informantes de los tres grupos de edad; y resultará interesante comprobar si, en el momento actual, el valenciano vive en Els Ports un proceso de sustitución lingüística, como se ha destacado con frecuencia o, por el contrario, ofrece signos de revalorización, especialmente por la ampliación de los usos de esta variedad a todos 
los ámbitos sociales, incluidos aquellos de naturaleza formal e institucional de los que tradicionalmente ha estado alejado.

De este modo, los 30 informantes por comunidad de habla que integran la muestra se distribuyen en cuotas idénticas en función del género (15 hombres y 15 mujeres) y la edad (10 jóvenes, 10 adultos y 10 mayores). Sin embargo, se concede el doble de informantes a los estratos medio (12) y bajo (12), que a la clase alta (6), con el fin de garantizar una representación más realista de la población analizada.

Estos 60 informantes fueron interrogados individualmente siguiendo el guion previsto en el cuestionario preparado a tal fin. Para evitar que los resultados quedaran sesgados por conocer la intencionalidad de la prueba, la información detallada sobre este estudio solo se ofreció a los informantes a posteriori. La prueba tuvo una duración media aproximada de 25 minutos y fue grabada mediante procedimientos audiovisuales por uno de los autores del estudio.

\subsection{Tratamiento de los resultados}

Para concluir las cuestiones metodológicas, debemos referirnos al tratamiento que hemos dado a todos estos datos. Hemos utilizado paralelamente los programas de cálculo estadístico SPSS 17.0 y Statgraphics Centurión 16. Las pruebas de significación se fundamentan en un test ANOVA, que arroja un $p$-valor que hemos tenido siempre en cuenta en el nivel inferior a 0,05. Por razones de claridad expositiva, omitimos en general dar cuenta de los correspondientes $p$-valores, y solo los traemos a colación en la medida en que resultan relevantes según el propósito de la investigación actual.

\section{Resultados y discusión}

Por lo que respecta a la primera de las preguntas, los resultados obtenidos en las dos comunidades de habla son los siguientes (vid. Tabla 1):

\begin{tabular}{|r|c|c|c|c|c|}
\cline { 2 - 5 } \multicolumn{1}{c|}{} & $\mathbf{1}$ & $\mathbf{2}$ & $\mathbf{3}$ & $\mathbf{4}$ & $\mathbf{5}$ \\
\hline ELS PORTS & $6 \%$ & $37 \%$ & $37 \%$ & $20 \%$ & $0 \%$ \\
\hline MATARRANYA & $0 \%$ & $7 \%$ & $37 \%$ & $37 \%$ & $20 \%$ \\
\hline
\end{tabular}

Tabla 1. ¿Es importante que se enseñe el catalán en las escuelas?

Como apreciamos en la tabla, en general, las respuestas más frecuentes en Els Ports se sitúan en los valores centrales de la tabla (un 37 \% para el sí no rotundo, un $37 \%$ para la respuesta neutra y un $20 \%$ para el no), lo cual ofrece una imagen muy equilibrada de las respuestas. En principio, y como veremos en las siguientes preguntas, podemos decir que se trata de respuestas poco arriesgadas (y que nos deben llevar a valorar en qué medida nuestros informantes han sido políticamente 
correctos o hasta qué punto para ellos esta cuestión no resulta especialmente candente, y de ahí su baja rotundidad). Por el contrario, en Matarranya, las respuestas son más claramente negativas (con un $57 \%$ de valores negativos, frente a un $37 \%$ de valores neutros y un escaso $7 \%$ de valores positivos).

En cuanto a la segunda de las preguntas, encontramos los datos correspondientes en la Tabla 2:

\begin{tabular}{|r|c|c|c|c|c|}
\cline { 2 - 6 } \multicolumn{1}{c|}{} & $\mathbf{1}$ & $\mathbf{2}$ & $\mathbf{3}$ & $\mathbf{4}$ & $\mathbf{5}$ \\
\hline ELS PORTS & $0 \%$ & $13 \%$ & $40 \%$ & $27 \%$ & $20 \%$ \\
\hline MATARRANYA & $0 \%$ & $13 \%$ & $0 \%$ & $40 \%$ & $47 \%$ \\
\hline
\end{tabular}

Tabla 2. ¿Es bueno que se enseñe en catalán en las escuelas y en los institutos?

La segunda pregunta resulta, en parte, de una precisión de la anterior, por cuanto abunda en ella, pero incidiendo en la posibilidad de que el catalán sea la lengua vehicular de la educación obligatoria. Recordemos que, en la comparación entre nuestras dos comunidades de habla, pues pone el foco directamente en la educación bilingüe $y$, como sabemos, la realidad a este respecto es sustancialmente diferente entre la Comunidad Valenciana y Aragón. Por un lado, en Els Ports la pregunta debe situarse en un debate acerca de cómo se aplica el modelo de educación bilingüe; por el contrario, en Aragón, donde la educación bilingüe no es una realidad, esta pregunta debe situarse en un contexto sumamente diferente. En cualquier caso, tanto en Els Ports como en Matarranya las respuestas a esta pregunta son sustancialmente diferentes de las anteriores. En la comarca castellonense, tan solo un $13 \%$ de respuestas claramente positivas (aunque con un $0 \%$ en la respuesta más rotunda, valor 1), y un más significativo $47 \%$ de respuestas negativas (un $20 \%$ de las cuales corresponden al valor 5, "en absoluto"). Por último, y ahora en consonancia con lo observado en las respuestas a la pregunta anterior, se documenta también un $40 \%$ de respuestas indeterminadas (valor 3). En principio, los datos parecen confirmar la idea anterior: hay muchos menos informantes que desean la escolarización en valenciano que la mera enseñaza del valenciano en el sistema escolar, como podría preverse ante la situación de diglosia leve que ya hemos referido para Els Ports. En cuanto a Matarranya, la opinión de la muestra acerca de la posibilidad de poner en marcha un modelo educativo bilingüe es mucho más contundente: solo un $13 \%$ de los informantes manifestaron estar a su favor, mientras que un llamativo 87 \% lo rechazaron.

La tercera y última pregunta del terno dedicado a la fidelidad lingüística y sus resultados se recogen a continuación (vid. Tabla 3):

\begin{tabular}{|r|c|c|c|c|c|}
\cline { 2 - 6 } \multicolumn{1}{c|}{} & $\mathbf{1}$ & $\mathbf{2}$ & $\mathbf{3}$ & $\mathbf{4}$ & $\mathbf{5}$ \\
\hline ELS PORTS & $0 \%$ & $7 \%$ & $30 \%$ & $40 \%$ & $23 \%$ \\
\hline MATARRANYA & $0 \%$ & $7 \%$ & $0 \%$ & $30 \%$ & $63 \%$ \\
\hline
\end{tabular}

Tabla 3. Y en la Universidad, ¿qué le/te parece que se enseñe en catalán? 
Esta última pregunta supone un grado más en la precisión: el último grado académico de formalidad siempre lo representan los estudios universitarios; $y$, por tanto, en el fondo estamos pidiendo la opinión de los informantes sobre que el valenciano sea lengua de uso universitario. Como puede comprobarse, en Els Ports el grueso de la muestra se ha decantado claramente por el no, con un $63 \%$ de las respuestas (valores 4 y 5), en oposición al escaso $7 \%$ que ha respondido que el sí (nótese también que el grado de rotundidad también está a favor de las respuestas negativas, pues el $23 \%$ ha respondido el valor 5 , mientras que nadie ha respondido con un sí rotundo, valor 1 ). ${ }^{3}$ Por su parte, en el mismo sentido parecen manifestarse los informantes de Matarranya, aunque de modo más rotundo: solo un $7 \%$ de respuestas favorables, frente a un $93 \%$ de respuestas negativas (y un $63 \%$ con el valor de máxima rotundidad).

A partir de los datos de estas tres preguntas, obtenemos el índice de fidelidad lingüística, que presentamos en la siguiente tabla (vid. Tabla 4). Este índice se obtiene agrupando los valores afirmativos, por un lado; y los negativos, por el otro. Después, hemos promediado las respuestas a las tres preguntas, de tal modo que obtenemos el porcentaje medio de respuestas afirmativas (antiguos valores 1 y 2 ), el de respuestas neutras (antiguo valor 3), y el de respuestas negativas (antiguos valores 4 y 5).

\begin{tabular}{|r|c|c|c|}
\cline { 2 - 4 } \multicolumn{1}{c|}{} & Sí & Ind. & No \\
\hline ELS PORTS & $21 \%$ & $36 \%$ & $43 \%$ \\
\hline MATARRANYA & $9 \%$ & $12 \%$ & $79 \%$ \\
\hline
\end{tabular}

Tabla 4. Fidelidad lingüística

Esto es, en Els Ports un $21 \%$ de los informantes se mostró decididamente partidario de la presencia del catalán en el sistema educativo, a un 36 \% le resultó indiferente, mientras que un 43 \% no la juzgó importante. Por su parte, en Matarranya, como se ve, solo un $9 \%$ de los informadores se mostró decididamente partidario de la presencia del catalán en el sistema educativo, frente a un $12 \%$ a quien le resultó indiferente, y un 79 \% que no la juzgó importante.

Hasta el momento, hemos comprobado como la diferente situación sociolingüística de ambas comunidades de habla se manifiesta con claridad en la diferente percepción que los informantes tienen acerca de la presencia de su lengua propia en el sistema educativo o, lo que es lo mismo, a la naturaleza bilingüe de un modelo educativo en el que su propia variedad goce de protagonismo. Sin embargo, si nos interesa calibrar en qué medida ello es fruto de las diferentes políticas lingüísticas puestas en marcha en la Comunidad Valenciana -o de su ausencia en Aragón-, debemos atender a la incidencia del factor generacional. Para que se confirmara nuestra hipótesis, los jóvenes de Els Ports tendrían que tener una actitud hacia la presencia del catalán en el sistema educativo no solo más favorable que los de Matarranya, sino también mejor que las de sus convecinos adultos y mayores. Veamos, pues, la Tabla 5: 


\begin{tabular}{|c|c|c|c|}
\multicolumn{1}{c|}{} & \multicolumn{3}{|c|}{ Els Ports } \\
\hline Sí & J & A & M \\
\hline 37 & 13 & 13 \\
\hline Ind. \\
\hline No \\
\hline 26 & 64 & 17 \\
\hline 37 & 23 & 70 \\
\hline
\end{tabular} \begin{tabular}{|c|c|c|c|}
\hline \multicolumn{3}{c|}{ Matarranya } \\
\hline 20 & 0 & 7 \\
\hline 17 & 13 & 7 \\
\hline 63 & 87 & 87 \\
\hline
\end{tabular}

Tabla 5. Fidelidad lingüística en Els Ports y en Matarranya (por grupos de edad)

Como vemos, en Els Ports las actitudes hacia la presencia del catalán en el sistema educativo no son estables, sino que son mucho mejores en los jóvenes que en los restantes informantes ( $y$, a su vez, son mejores en los adultos que en los mayores, como prueba de que las políticas lingüísticas, y entre ellas las educativas, fructifican tanto más cuanto más pasa el tiempo). Así, con los valores destacados en cursiva en la tabla podemos intuir esa transición desde los valores predominantemente desfavorables de los mayores, a la actitud más neutra de los adultos y a picos de actitud favorable nada desdeñables en los jóvenes (la diferencia es estadísticamente significativa). Por el contrario, en Matarranya la situación es muy distinta. Es cierto que los jóvenes presentan actitudes mucho más favorables que el resto de la muestra, pero abundan, en cualquier grupo generacional, las actitudes negativas. $Y$ las diferencias entre los jóvenes y los demás informantes son demasiado leves para tomarlas en consideración (la diferencia no es estadísticamente significativa).

\section{Conclusiones}

Tras la exposición de estos datos, y a partir de algunas de las valoraciones con que hemos ido acompañando dicha exposición, es fácil determinar que nuestras conclusiones van en dos claras líneas. La primera, puramente sociolingüística, nos lleva a concluir que, en Els Ports y en Matarranya, la situación sociolingüística apunta a una clara diglosia, como por otra parte ya intuíamos. Y esa diglosia, sin duda, es sustancialmente diferente en una y otra comarca, como respuesta a contextos legislativos y educativos diferentes. La segunda, por su parte, señala a la propia actitud de la población de estas dos comunidades de habla con respecto de la presencia de la lengua propia en el sistema educativo, a un tiempo causa y consecuencia parcial de la situación que acabamos de describir. Veamos todo ello con cierto detenimiento.

Al evaluar las diferencias entre los respectivos índices de fidelidad lingüística de las comarcas de Els Ports y Matarranya, no hay duda de que nos encontramos ante comunidades en las que el español sigue desplazando al catalán de los usos prestigiados. Eso se traduce en niveles bajos de respuestas afirmativas a las preguntas acerca de la presencia del catalán en los distintos niveles educativos, como se ve. Con todo, en ningún caso puede afirmarse que la situación de Els Ports y la de Matarranya sean análogas, pues esa diglosia es mucho más acusada en la comarca aragonesa, en la que los índices son muy inferiores. Para corroborar esta impresión, 
bastará cotejarla con los índices de orgullo lingüístico y presión social subjetiva que también hemos documentado en estas comarcas, en el transcurso de otras investigaciones, y que referimos sucintamente a continuación (pueden ampliarse en González Martínez (2010, 2011)):

\begin{tabular}{|r|c|c|c|}
\cline { 2 - 4 } \multicolumn{1}{c|}{} & Sí & Ind. & No \\
\hline ELS PORTS & $20 \%$ & $23 \%$ & $57 \%$ \\
\hline MATARRANYA & $0 \%$ & $17 \%$ & $83 \%$ \\
\hline
\end{tabular}

Tabla 6. Orgullo lingüístico

\begin{tabular}{|r|c|c|c|}
\cline { 2 - 4 } \multicolumn{1}{c|}{} & Sí & Ind. & No \\
\hline ELS PORTS & $18 \%$ & $43 \%$ & $39 \%$ \\
\hline MATARRANYA & $4 \%$ & $13 \%$ & $83 \%$ \\
\hline
\end{tabular}

Tabla 7. Presión social subjetiva

No hay duda de que estos indicadores confirman lo que ya habíamos anunciado: bajos porcentajes de respuestas positivas, altos en las negativas: señal inequívoca de que los informantes tienen una mejor consideración del español que del catalán en lo que respecta a su uso en situaciones formales, en este caso la educación. Algo que, por otra parte, se ha venido confirmando en anteriores estudios, tanto en la Comunidad Valenciana (Ros, 1980; Blas Arroyo 1994, 1995, 1997; Safont, 2007, etc.) como en la Franja Oriental de Aragón (Martín Zorraquino, 1995).

Este primer diagnóstico coincide plenamente con lo que habíamos diagnosticado también por medio de técnicas indirectas como el matched-guise en ambas comarcas: en Els Ports y en Matarranya los informantes conceden mayor valor instrumental al español que al catalán, mientras que la variedad propia continúa recibiendo los mejores valores en la esfera personal (González Martínez y Blas Arroyo, 2011 y 2012). Asimismo, el diagnóstico general es compartido, pero no la intensidad en el grado de esa diglosia o conflicto lingüístico; y así, se confirma que es mucho menos intensa en Els Ports que en Matarranya, como respuesta a las políticas lingüísticas -y entre ellas, sobre todo las educativas - operadas en la Comunidad Valenciana que no se han conocido en Aragón.

A la sazón, todo ello adquiere mayor coherencia, como hemos visto, al atender al componente dinámico que muestra en Els Ports -y no en Matarranya- el análisis del factor generacional en esas actitudes, que demuestra que son mucho más positivas en los jóvenes que en los adultos o mayores. 
Ahora bien, la otra cara de esta misma moneda es la que atañe a las actitudes acerca de la educación bilingüe específicamente. No en vano, debemos proponer una breve reflexión acerca de la importancia del sistema educativo y de su especial incidencia en el statu quo que acabamos de describir, como respuesta al principal objetivo de nuestra reflexión de hoy. Una vez constatado el dinamismo que proponemos en la situación sociolingüística diagnosticada en Els Ports, debemos preguntarnos por las causas que han conducido a esa situación, más favorable al valenciano en tiempos recientes. Una respuesta simple apuntaría hacia el proceso de normalización que arranca hace casi ya tres décadas con la promulgación de la Llei d'ensenyament $i$ ús del valencià de 1983. A ella remiten la mayoría de los autores citados que han trabajado en las actitudes lingüísticas en las dos últimas décadas. Ahora bien, creemos que una causa de primer orden en ese estado de cosas es, sin duda, la acción educativa. Junto a los resultados del proceso de normalización lingüística en otras esferas sociales, pero probablemente por encima de todas ellas, las principales diferencias actitudinales que hemos observado entre Els Ports y Matarranya se derivan en buena medida del diferente tratamiento de que es objeto la lengua propia en sus respectivos sistemas educativos. Y de ahí que donde más diferencias hayamos obtenido sea precisamente entre los informantes jóvenes de cada comarca. Los de Els Ports asisten con regularidad a clases en valenciano, y tienen la obligación de conocer su lengua propia; los de Matarranya tienen solo la posibilidad -a veces peregrina- de estudiar su lengua, y en ningún caso ven reconocido su derecho a estudiarla.

En este sentido, al analizar a los escolares del Baix Cinca, en la aragonesa Franja de Ponent, Huguet y Biscarri (1995) ha visto la asociación directa entre actitudes lingüísticas positivas hacia el catalán y la asistencia a clase en esta lengua. Asimismo, este autor (Huguet, 2006) certifica las diferencias entre las actitudes lingüísticas de los escolares del Baix Cinca que asisten a clases optativas de catalán y las de aquellos que no lo hacen. De hecho, Huguet (2006: 153) asimila las actitudes positivas hacia el catalán de los alumnos de Baix Cinca que asisten a clase de catalán regularmente con los de la vecina comarca ilerdense Baix Segre, que sistemáticamente se escolarizan en esa lengua. $\mathrm{Ni}$ que decir tiene que este mismo autor ha venido demostrando la directa relación existente entre educación bilingüe y actitudes lingüísticas, y no solo hacia la lengua propia, sino también hacia las extranjeras (Huguet, 2008). Sin duda, por lo que se desprende de los estudios de este autor, la escolarización en catalán en el Baix Cinca es más sistemática que en Matarranya, donde la oferta ni es regular para todos los cursos ni siquiera todos los años; a ello, con seguridad, se añaden cuestiones de identidad cultural (la comarcas septentrionales de la Franja Oriental de Aragón están más unidas a Cataluña que Matarranya, que mira más a Teruel).

Llegados a este punto, debemos confirmar las conclusiones de Fernández Ulloa (2003) en su estudio sobre la educación bilingüe en el País Vasco. Superado el primer escollo del reconocimiento legal, necesario para garantizar la presencia de una lengua en todos los ámbitos sociales -también, por supuesto, la educación-, esta autora concluye sin dudas que la escolarización en la lengua minoritaria es necesaria 
e imprescindible; pero no suficiente. En su caso, reflexiona acerca del escaso poder que ha tenido la escolarización en vasco para revertir la lengua habitual de los intercambios en actividades escolares no monitorizadas por el docente (ya no hablamos siquiera de los usos personales). Acaso podríamos compartir esa reflexión para muchos territorios de la Comunidad Valenciana, incluso para Cataluña; pero qué duda cabe de que en el caso de Aragón todavía estamos en el paso anterior, el de garantizar que el catalán esté presente en el sistema educativo de pleno derecho.

La diferencia de actitudes hacia la escuela bilingüe entre Els Ports y Matarranya, sin duda, pone el dedo en la llaga de esa diferente realidad. Esas actitudes son mucho menos favorables en Matarranya por distintos factores; pero es evidente que uno de ellos, quizá el más importante, es que juzgan algo que no se produce y que, por tanto, no se siente natural. ${ }^{4}$ En Els Ports, los informantes conocen -y quizá de primera mano- los modelos bilingües $y$, por tanto, sus opiniones son por fuerza menos negativas. Al fin, nos encontramos en un círculo que debemos romper: la ausencia de una verdadera educación bilingüe en los territorios bilingües de Aragón hace que las actitudes hacia ella sean poco favorables; $y$, con esas actitudes, difícilmente una comunidad de habla entenderá motu proprio cuán importante es dicho modelo bilingüe para garantizar la conservación de su propio patrimonio lingüístico y cultural.

\section{Notas}

${ }^{1}$ Promulgada en 2009, la Ley de Lenguas de Aragón fue la respuesta legislativa a la histórica demanda de los territorios aragoneses bilingües de que sus variedades propias gozaran de reconocimiento oficial. La citada ley, por supuesto, reconoce explícitamente la necesidad de que estas lenguas minoritarias -catalán y aragonésgocen de protección y promoción a cuenta de las arcas autonómicas. Sin embargo, hasta el momento de escribir estas letras, tales preámbulos no habían conocido el desarrollo y la concreción necesarias; y todo a punta a que no lleguen a conocerlo por el momento, pues el nuevo Gobierno autonómico, surgido de las urnas en el pasado mayo de 2011, ya ha anunciado la derogación de la polémica Ley de Lenguas de Aragón de 2009.

${ }^{2}$ Este cuestionario sociolingüístico forma parte de una investigación de más alcance en la que, en el contexto de una entrevista sociolingüística semidirigida, se intercalaban técnicas directas (el cuestionario que nos ocupa) e indirectas (un cuestionario matched-guise y otro de gramaticalidad/aceptabilidad). Aun conscientes de la prevalencia de las técnicas indirectas para el estudio de las actitudes lingüísticas, hemos considerado oportuno dedicar este espacio a los datos obtenidos mediante el cuestionario sociolingüístico, no solo por el interés intrínseco de estos, sino también porque permiten una aproximación a opiniones y creencias muy reveladora acerca del contexto sociolingüístico en el que se desenvuelve en la actualidad la comarca de Els Ports. En el caso concreto de nuestra presente reflexión, 
no hay duda de que nos permiten aproximarnos a la realidad sociolingüística de las dos comunidades, y a sus opiniones sobre sus modelos educativos, de un modo que las técnicas indirectas no nos permiten.

3 Queremos dejar constancia de la incomodidad de muchos informantes ante su propia respuesta negativa, especialmente cuando se utilizaba el valor de máxima rotundidad; de hecho, en muchos casos los informantes parece que debieron de sentirse obligados a justificarse, con apelaciones comunes a a la mayor utilidad lingüística del español, por su mayor proyección nacional e internacional. En la mayoría de los casos, la justificación apeló a cuestiones de utilidad lingüística y de proyección del alumno fuera del ámbito regional, con independencia de su edad y de su estrato social. En el caso de Matarranya, las justificaciones fueron mucho menos frecuentes pero, cuando aparecieron en el discurso, apuntaban en el mismo sentido.

${ }^{4}$ Pasamos de puntillas sobre esta cuestión, pero no sin evidenciar la complejidad de un asunto en el que intervienen aspectos como las diferentes perspectivas que ofrecen las distintas opciones políticas, las opuestas voluntades de construcción de la identidad comarcal, regional y nacional, la contraria concepción que tienen algunas partes de la comunidad de habla sobre la filiación lingüística de la variedad propia, en relación directa con la cuestión de la unidad lingüística del catalán, etc.

\section{Referencias bibliográficas}

ACADÈMIA VALENCIANA DE LA LLENGUA (2004) Llibre blanc de l'ús del valencià. Valencia. AVL..

ARNAU, J. (1992) Educación bilingüe: modelos y principios psicopedagógicos. ARNAU, J.; COMET, C.; SERRA, J. M. and VILA. I. (Eds.): La educación bilingüe, 11-51. Barcelona: ICE/Horsori.

BLAS ARROYO, J. L. (1994) Valenciano y castellano. Actitudes lingüísticas de la sociedad valenciana. Estudio sobre una comunidad urbana. Hispania, 77, 1. 143-155.

BLAS ARROYO, J. L. (1995) De nuevo el español y el catalán, juntos y en contraste. Estudio de actitudes lingüísticas. Sintagma, 7, 29-41.

BLAS ARROYO, J. L. (1997) Dimensiones sociolingüísticas del cambio de código. A propósito de un corpus radiofónico. ITL Review of Applied Linguistics, 117-150.

FERNÁNDEZ-ULLOA, T. (2003) La Educación Bilingüe en el País Vasco: Problemas y Retos. Proceedings of the 4th International Symposium of Bilingualism. Sommerville, MA: Cascadilla Press. 703-729.

FERRER, F. (2000) Languages, Minorities and Education in Spain: The Case of Catalonia. Comparative Education, Vol. 36, No. 2, 187-197. 
FISHMAN, J. A. (1967) Bilingualism with and without diglossia, diglossia with and without bilingualism, Journal of Social Issues, 32: 29-38.

FISHMAN, J. A. (1972) La sociología del lenguaje, Madrid, Alianza (trad. española 1982).

FISHMAN, J. A. (1977) The sociology of bilingual education. Spolsky, B. and Cooper, R. L. (eds). Frontiers of Bilingual Education. Rowley, MA: Newbury House.

FISHMAN, J. A. (1991) Reversing language shift. Theoretical and empirical foundations of assistance to threatened languages. Clevedon: Multilingual Matters Ltd.

GENERALITAT DE CATALUNYA (2004) Enquesta d'usos lingüístics a la Franja d'Aragó 2004. Dades sintètiques, Barcelona, Secretaria General de Política Lingüística.

GENESEE, F. (2006) Una revisió dels programes d'immersió en francès al Canadà. GENERALITAT DE CATALUNYA (Ed.) El coneixement de les llengües a Catalunya, 56-77. Barcelona: Departament d'Educació i Universitats.

GENESEE, F.; LAMBERT, W. E.; and HOLOBOW, N. E. (1986) La adquisición de una segunda lengua mediante inmersión: el enfoque canadiense. Infancia y Aprendizaje, 33. 27-36.

GONZÁLEZ MARTÍNEZ, J. (2009) Actitudes lingüísticas en Els Ports (Castellón) y Matarranya (Teruel). UNED. Tesis doctoral

GONZÁLEZ MARTÍNEZ, J. (2010) Actitudes lingüísticas en una comunidad rural: Els Ports (Castellón). Datos de un cuestionario sociolingüístico. Cultura, lenguaje y representación, 8: 75-95.

GONZÁLEZ MARTÍNEZ, J. (2011) Actitudes lingüísticas en Matarranya (Teruel, España). Datos de un cuestionario sociolingüístico.» Estudios de Lingüística Aplicada (México), 52.

GONZÁLEZ MARTÍNEZ, J.; BLAS ARROYO, J. L. (2011) «Estabilidad y dinamismo en las actitudes lingüísticas de una comunidad bilingüe española (Els Ports, Castellón).Hispania. A Journal Devoted to the Teaching of Spanish and Portuguese, 94(4): 663-679.

GONZÁLEZ MARTÍNEZ, J.; BLAS ARROYO, J. L. (2012) Patterns of change and continuity in the language attitudes of several generations in two bilingual Spanish communities: the rural regions of Els Ports and Matarranya, International Journal of Bilingual Education and Bilingualism, 15:2, 199-215

HUGUET, Á. (2006) Attitudes and motivation versus language achievement in crosslinguistic settings. What is cause and what effect? Journal of Multilingual \& Multicultural Development, 27(5), 413-429.

HUGUET, Á. (2007) Minority languages and curriculum. The case of Spain. Language. Culture \& Curriculum, 20 (1), 70-86. 
HUGUET, Á. et al. (2008) La educación bilingüe: ¿Una respuesta al Aragón trilingüe? Revista de Psicodidáctica, 13. N. 2. 13-31.

HUGUET, Á. y BISCARRI, J. (1995) Actitudes lingüísticas de los escolares en el Baix Cinca. Incidencia de algunos factores. Revista Interuniversitaria de Formación del Profesorado, 23. 163-175.

HUGUET, Á. y E. LLURDA (2001) Language attitudes of School Chindren in Two Catalan/Spanish Bilingual Communities. Internacional Journal of Bilingual Education and Bilingualism, 4, 267-282.

HUGUET, Á.; LAPRESTA, C. y MADARIAGA, J. M. (2008) A study on language attitudes towards regional and foreign languages by school children in Aragon (Spain). International Journal of Multilingualism, 5(4), 275-293.

LAMBERT, W. E. (1974) Culture and language as factors in learning and education. ABOUD, F. and Meade, R. D. (Eds.): Cultural factors in learning and education, 91-122. Bellinghan: Western Washington State College.

LAMBERT, W. E. (1981) Un experimento canadiense sobre desarrollo de competencia bilingüe. Programa de cambio de lengua hogar-escuela. Revista de Educación, 268. 167-177.

LAPRESTA, C.; HUGUET, Á.; JANÉS, J. (2010) Análisis discursivo de las actitudes lingüísticas de los escolares de origen inmigrante en Cataluña. Revista de Educación, 353. Septiembre-Diciembre 2010, pp. 521-547.

LINDHOLM-LEARY, K. (2006) Resultats de I'alumnat que participa en programes de Ilengua dual als Estats Units. GENERALITAT DE CATALUNYA (Ed.), El coneixement de les llengües a Catalunya, 78-94. Barcelona: Departament d'Educació i Universitats.

LÓPEZ MORALES, H. (1994) Metodología de la investigación lingüística, Salamanca, Ediciones Colegio de España.

LÓPEZ MORALES, H. (2004) Sociolingüística, Madrid, Gredos, 1989, tercera edición, aumentada.

MACKEY, W. F. (1970) A tipology of bilingual education. Foreign Language Annals, 34. 596-608.

MACKEY, W. F. (1976) Bilinguisme et contact dans langues. Paris: Kilincksieck.

MARTÍN ZORRAQUINO. M. A. (1995) Estudio sociolingüístico de la Franja Oriental de Aragón. Zaragoza. Universidad de Zaragoza.

MESTRE, J. (1991) Cataluña. Situación actual del bilingüistmo en la enseñanza en Cataluña. Revista de Educación, 268. 203-252.

RENDON, S. (2007) The Catalan premium: language and employment in Catalonia. Journal of Population Economics, Vol. 20, No. 3, 669-686. 


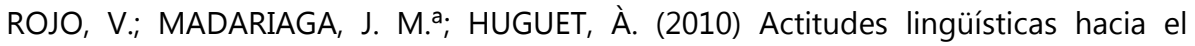
euskera y castellano de los estudiantes autóctonos e inmigrantes de la Educación Secundaria Obligatoria. Cultura y Educación, 22(1), 85-97.

ROS. M. (1982) Percepción y evaluación de los hablantes de cinco variedades lingüísticas. NINYOLES, R.: Estructura social al País Valencià. Valencia. Diputación Provincial. 679-698.

SAFONT, M. P. (2007) Language Attitudes and Language Use in the Valencian Community. In LASAGABASTER, D. and HUGUET, Á. (eds.).: Multilingualism in European bilingual contexts: Language use

SERRA, J. M. (2006) El plan de lengua y cohesión social en Cataluña: primeros datos de una investigación. Cultura y Educación,18 (2), 159-172.

SKUTNABB-KANGAS, T. (1981) Bilingualism or Not: The Education of Minorities. Clevedon: Multilingual Matters.

SKUTNABB-KANGAS, T. (1988) Multilingualism and the education of minority children. SKUTNABB-KANGAS T. and CUMMINS, J. (Eds.) Minority Education. From Shame to Struggle, 1-44. Clevedon: Multilingual Matters.

TORRES-GUZMÁN, M. E \& ETXEBERRÍA, F. (2005) Modelo B/Dual Language Programmes in the Basque Country and the USA. International Journal of Bilingual Education and Bilingualism, 8(6), 506-528

VILA, I. (1990) La normalització lingüística a l'ensenyament no universitari de Catalunya (1980-1990), Treballs de sociolingüística catalana, 9. 47-73.

VILA, I. (1995) El català i el castellà en el sistema educatiu de Catalunya. Barcelona: Horsori.

VILA, I. (ed) (1998) Bilingüisme i Educació. Barcelona: Proa.

WEINREICH, U. (1953) Lenguas en contacto. Descubrimientos y problemas, Caracas, Universidad Central (trad. española 1974).

\section{Nota biogràfica}

Juan González Martínez. Doctor en Filologia. Licenciado en Antropología Social y Cultural. Máster en Tecnología Educativa. Profesor del Departament de Pedagogia de la Universitat Rovira i Virgili. Centra sus intereses en las actitudes lingüísticas en contextos de conflicto lingüístico, así como en el uso de la tecnología en diferentes contextos educativos.

Universitat Rovira i Virgili. Departament de Pedagogia. Campus Terres de I'Ebre. Avda. de Remolins, 13-15. 43500 Tortosa (Tarragona)

Correo-e.: juan.gonzalezm@urv.cat 